Z Rheumatol $2017 \cdot 76: 836-837$

https://doi.org/10.1007/s00393-017-0398-x

Online publiziert: 27. November 2017

C) Springer Medizin Verlag GmbH 2017

CrossMark

\author{
B. Swoboda' $\cdot$ W. Rüther ${ }^{2}$ \\ ' Orthopäd.-Rheumatolog. Abteilung, Orthopäd. Univ.-Klinik, Waldkrankenhaus St. Marien, Erlangen, \\ Deutschland \\ ${ }^{2}$ Klinik und Poliklinik für Orthopädie, Universitätsklinikum Hamburg-Eppendorf (UKE), Hamburg, \\ Deutschland
}

\title{
Rheuma und Wirbelsäule
}

Bei diesem Thema wird der klinisch tätige Rheumatologe bei der rheumatoiden Arthritis (RA) sofort an Instabilitäten der Kopfgelenke und bei der ankylosierenden Spondylitis (Morbus Bechterew) an die Frage aufrichtender Operationen (Kolumnotomien) bei in Kyphose eingesteifter Wirbelsäule denken. Auch wenn in der Ära der Biologikatherapie schwere destruierende Veränderungen der Kopfgelenke bei RA seltener geworden sind, darf ein aktuelles Statement zur adäquaten Diagnostik und operativen Therapie nicht fehlen. Gleiches gilt für aufrichtende Operationen beim Morbus Bechterew. Diese beiden Themen sind auch für internistische Kollegen von Relevanz, um zeitgerechte operative Interventionen nicht $\mathrm{zu}$ versäumen und Patienten adäquat beraten zu können, wenn sie nach ihrer Zweitmeinung gefragt werden.

\section{》) Das klinische Bild der rheumatoiden Arthritis wirft v. a. im Alter komplexe Fragestellungen auf}

Unabhängig von diesen beiden Themenkomplexen wirft das klinische Bild der rheumatoiden Arthritis v. a. im Alter komplexe Fragestellungen auf. Allgemeinorthopädische und auch geriatrische Probleme, wie z. B. die Osteoporose mit Wirbelkörpersinterungen, werden bei der RA durch die Krankheit selbst und die oft jahrelange Kortisontherapie akzentuiert, sodass hierbei insbesondere bei dem älter werdenden Rheumapatienten die Orthesenversorgung der osteoporotischen Wirbelsäule mit Wirbelkörpersinterungen immer mehr an
Bedeutung gewinnt. Die oft vernachlässigte und häufig auch bagatellisierte Orthesenversorgung der osteoporotischen Wirbelsäule stellt jedoch in Bezug auf Indikation, Anpassen der Orthese sowie Kontrolle der Passform eine anspruchsvolle ärztliche Tätigkeit aus dem Bereich der Orthopädietechnik dar. Während traditionelle starre Orthesen sich negativ auf Muskelkraft und -masse auswirken, sind bei osteoporotischen Wirbelkörperverformungen flexible Rucksackorthesen besser geeignet, die funktionellen Kapazitäten Betroffener zu verbessern. Kenntnisse hierzu sind die Voraussetzung, um Patienten vom Nutzen derartiger Orthesen $\mathrm{zu}$ überzeugen, sodass diese getragen werden und nicht in der Abstellkammer landen. Zweifelsfrei befreit die Rezeptur einer Orthese nicht von weiteren Maßnahmen der physikalischen Therapie und insbesondere der Kräftigung der rumpfstabilisierenden Muskulatur.

Die Lendenwirbelsäule hat bei RA immer verhältnismäßig wenig Beachtung gefunden. Spezifische Veränderungen wie an der rheumatischen Halswirbelsäule fehlen, und auch im Vergleich $\mathrm{zu}$ rein degenerativen Veränderungen sind an der Lendenwirbelsäule keine für eine RA charakteristischen Merkmale nachweisbar. Klinische Studien haben eine hohe Inzidenz von Lendenwirbelsäulenbeschwerden dokumentiert. Die Schmerzintensität korreliert mit der Entzündungsaktivität, und die Progredienz einer De-novo-Skoliose bei Instabilität kann rasch sein. Schmerzen an der Lendenwirbelsäule dürfen bei Rheumapatienten nicht weiter vernachlässigt werden, sondern erfordern eine detaillierte Analyse, um einen adäqua- 
ten Therapieplan bis hin zur operativen Intervention $\mathrm{zu}$ erstellen.

Abgerundet wird dieses Schwerpunktheft durch einen Beitrag zur Bildgebung der Sakroiliakalgelenke, da insbesondere magnetresonanztomographische Befunde die Grundlage für das Konzept der nichtradiologischen axialen Spondylitis neben der klassischen ankylosierenden Spondylitis darstellen.

\section{Fazit}

Die Behandlung des Rheumapatienten stellt nach wie vor eine interdisziplinäre Herausforderung dar. Die Beiträge dieses Schwerpunktheftes zeigen zweifelsfrei, dass Probleme an der Wirbelsäule nicht allein durch eine adäquate Basistherapie oder auch Schmerztherapie gelöst werden können, sondern im Rahmen umfassender Therapiestrategien Erfahrungen und Kenntnisse aus anderen Bereichen, wie z. B. orthopädischer Rheumatologie, physikalischer, technischer Orthopädie und Wirbelsäulenchirurgie, erfordern.

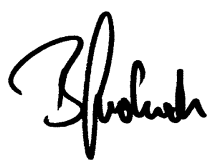

B. Swoboda

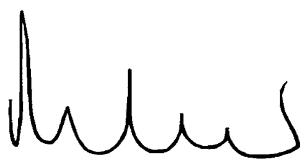

W. Rüther

\section{Korrespondenzadresse \\ Rheuma - weniger Medika- mente, mehr Lebensqualität?}

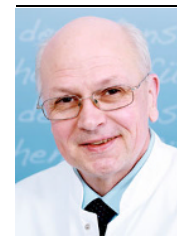

Univ.-Prof. Dr. B. Swoboda

Orthopäd.-Rheumatolog.

Abteilung, Orthopäd. Univ.-

Klinik, Waldkrankenhaus St.

Marien

Rathsberger Str. 57, 91054 Erlangen, Deutschland bernd.swoboda@ waldkrankenhaus.de

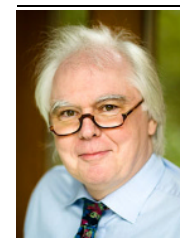

Prof. Dr. W. Rüther

Klinik und Poliklinik für Orthopädie, Universitätsklinikum Hamburg-Eppendorf (UKE) Martinistraße 52, 20251 Hamburg, Deutschland ruether@uke.de

Interessenkonflikt. B. Swoboda und W. Rüther geben an, dass kein Interessenkonflikt besteht.

Sind Patienten über längere Zeit beschwerdefrei, wünschen sie sich oft, auf Medikamente verzichten zu weisen darauf hin, dass ein kontrollität führen könne.

"Den Erfolg einer Therapie empfinden lichen Erkrankungen wie einen NeuanLorenz, Präsident der DGRh. „Dennoch erscheint der Wunsch, mit möglichst wenig Medikamenten auszukommen, sehr können. Experten der Deutschen Gesellschaft für Rheumatologie (DGRh) liertes Absetzen zu mehr Lebensquaviele Patienten mit rheumatisch-entzündfang", berichtet Professor Dr. Hanns-Martin nachvollziehbar", so der Leiter der Sektion Rheumatologie am Universitätsklinikum Heidelberg. Denn die Therapie kann auch unerwünschte Nebenwirkungen nach sich ziehen, wie Magen-Darm-Beschwerden, Hautreizungen oder Kurzatmigkeit. Studien haben gezeigt: Je kürzer ein Patient erkrankt war, desto größer die Chancen für einen erfolgreichen Therapieabbau. Es ist aussichtsreicher, lediglich die Dosis zu verringern, als die Präparate ganz abzusetzen. „Wann, bei wem und wie ein Therapieabbau durchgeführt werden kann, müssen Arzt und Patient im Gespräch klären", betont Lorenz. Als Voraussetzung gilt, dass der Patient mindestens sechs Monate in Remission war. Wichtig sei zudem eine engmaschige Kontrolle. „Die Patienten müssen gemeinsam mit dem Rheumatologen abwägen, was die Lebensqualität stärker einschränkt: die Erkrankung oder die Nebenwirkungen", so Lorenz.

Wirtschaftliche Überlegungen oder gar das Drängen seitens der Krankenkasse wie es beim Einsatz kostspieliger Biologika schon vorkam - dürfen keinen Einfluss auf die Entscheidung haben. Entscheidend sei: „Das Reduzieren der Rheuma-Medikamente darf ausschließlich der Lebensqualität des Patienten dienen."

Literatur: Haschka J et al. Ann Rheum Dis (2016) 75:45-51; doi: 10.1136/annrheumdis-2014-206439.

Pressestelle, Deutsche Gesellschaft für Rheumatologie (DGRh) 